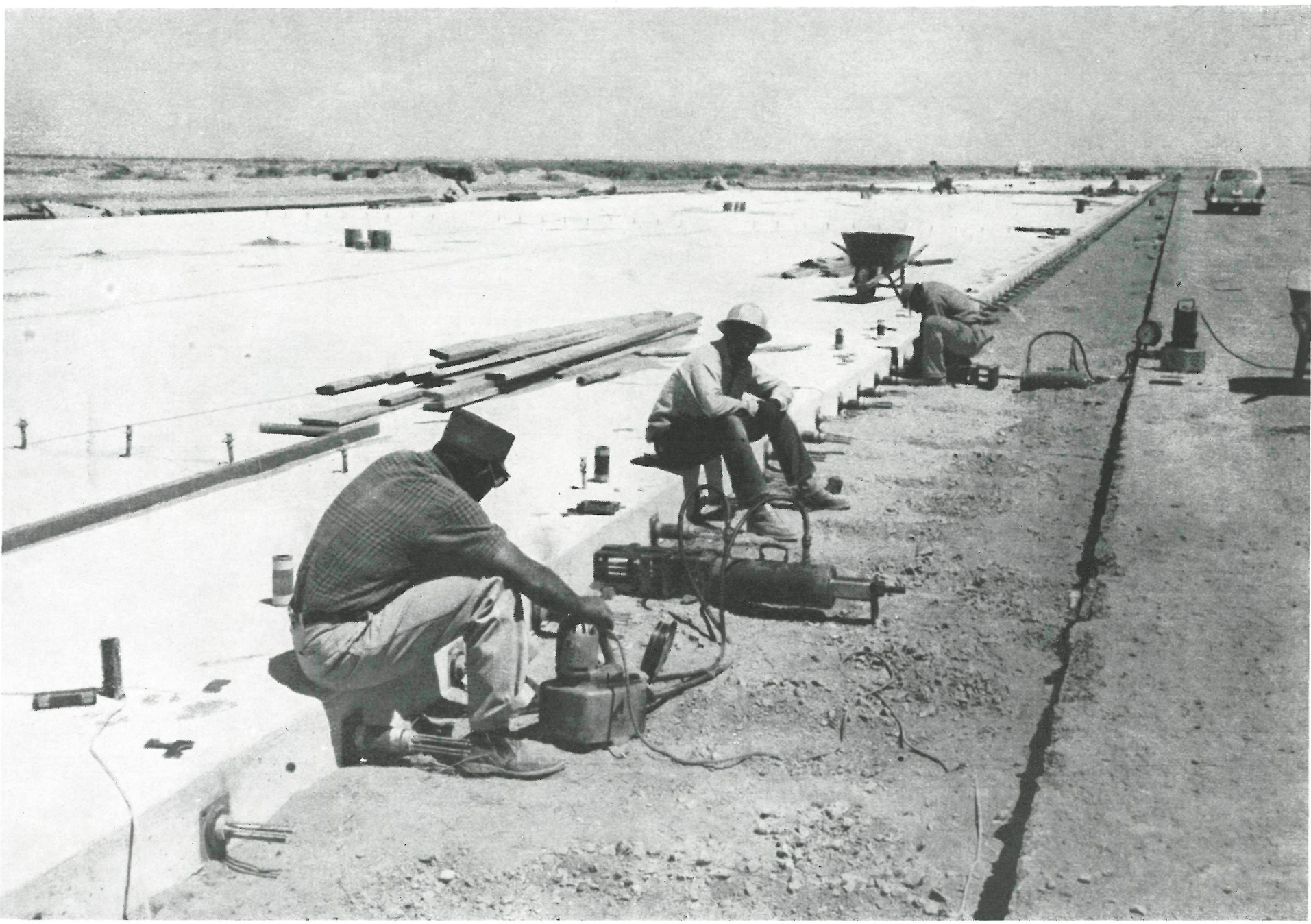

$514-41$

\title{
pistas de rodadura pretensadas
}

La primera pavimentación de hormigón pretensado se terminó en julio de 1959. Es una pista de vuelo, construída por el Corps of Engineers en la base aérea de Biggs, Texas (EE. UU.).

El proyecto se redactó bajo la dirección del coronel Albert $L$. Reed, ingeniero del Distrito de Albuquerque de Nuevo México. Este proyecto consiste en un refuerzo de pistas de vuelo y rodadura en la base antes mencionada, con objeto de poderlas capacitar para las maniobras de los bombarderos B. 52 que han sido asignados a la base.

El pavimento pretensado tiene un espesor de $23 \mathrm{~cm}, 22,8 \mathrm{~m}$ de anchura y $472 \mathrm{~m}$ de longitud en la pista de rodadura T.3. Parte de esta pista tiene $48 \mathrm{~cm}$ de espesor, con hormigón armado, y parte, de $60 \mathrm{~cm}$ de espesor, con hormigón en masa. Estos tres tipos de pavimentación se apoyan en una base, cuyo módulo es de $14 \mathrm{~kg} / \mathrm{cm}^{2}$. 


\section{Datos para el cálculo}

Las normas básicas para la redacción del proyecto se dictaron por el Corps of Engineers, partiendo de ló datos iniciales obtenidos de ensayos realizados en los laboratorios de Mariemont (Ohio), iniciados en diciembre de 1953

En este estudio se revisaron las distintas teorías sobre la técnica del hormigón pretensado y construcciones similares realizadas en otros paises E1 laboratorio realizó ensayos sobre modelos, y a la escala natural, de pavimentaciones de hormigón pretensado, con la finalidad de obtener un criterio general necesario antes de llevar a la realización las obras que debían ejecutarse. planta y alzado
de la pista
1. Espaldón asfáltico.--2. Arco de espaldón de $15 \mathrm{~m} .-3$. Hormigón pobre.-4. Junta de construcción.- . Pseudo-junta.-6. Eje de la pista.-7. Junta de dilatación.-8. Diez centímetros.-9. Quince centíme.
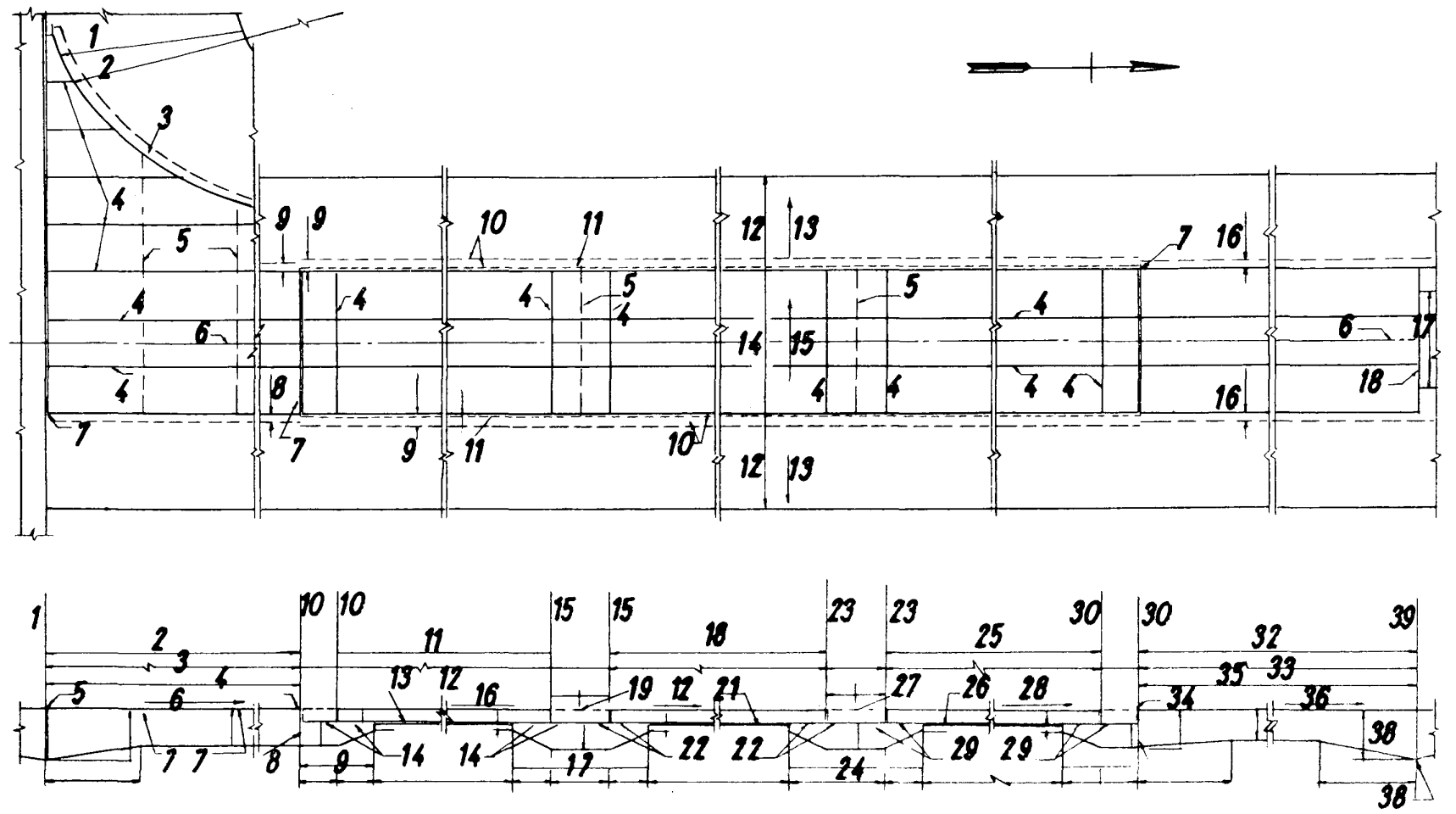

1. Estación de replanteo.-2. Primera parte (hormigón ordinario). -3. Ciento sesenta y siete metros.-4. Pseudo-juntas espaciadas a 7,62 m.-5. Junta de dilatación.-6. Pendiente 0,30\%.-7. Pseudo-juntas.-8. Pasadores.-9. Viga de hormigón armado.-10. Estación de replante. parte (pretensada) - 19. Pseudo-junta - 20. Papel sobre epante arena - 21. Pendiente 0.20\%.-22. Papel de construcción.-23. Estación parte (pantionte $0,20 \%$. - 22. Papel de construccion.-23. Estación 28. replanteo.-24. Viga de hormigon armado--25. Cuarta parte (pretensada).-26. Ciento cincuenta y dos metros.-27. Pseudo-junta. ta parte (hormigón armado - -33. Cuatrocientos cincuenta y siete metros.-34. Pseudo-juntas espaciadas a 7,60 m.-35. Junta especial de dilatación.-36. Pendiente variable.-37. Pasadores.-38. Borde recrecido.-39. Estación de replanteo.

Al igual que otros pavimentos, los de hormigón pretensado se pueden considerar como una estructura colocada sobre una base cuya capacidad de sustentación tiene un valor determinado. Sin embargo, contrariamente a lo que ocurre en otros tipos, el pavimento pretensado se proyecta de tal forma que permite un agrietamiento en el fondo del pavimento al someterlo a ciertas cargas, puesto que continúa su función como una sola unidad después de haberse formado esas grietas.

Estas características son posibles gracias al control $\epsilon$ fectivo del pretensado sobre la profundidad del agrietamiento o fisuración formada $\mathrm{y}$, esencialmente, por cerrar las grietas después de desaparecer la carga que las originó. A este respecto, los efectos nocivos que estas fisuraciones pudieran producir en otras clases de pavimentación desaparecen todas en este tipo particular.

Las grietas, actuando como articulaciones plásticas redistribuyen los momentos debidos a la carga, y permiten pavimentar con menores espesores de los que habría de asignarse de no emplearse el tipo pretensado. Debido a esta reducción de espesor, los esfuerzos originados en el alabeo provocado por cambios de temperatura, también se ven reducidos, y se pueden compensar aumentando el esfuerzo de pretensado necesario para las cargas dinámicas. Puesto que el esfuerzo debido al rozamiento en el contacto con la base también se puede compensar introduciendo un esfuerzo adicional de pretensado, se pueden construir trozos de pavimentación de mayor longi-

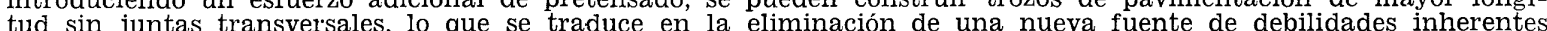
tud sin juntas transversales, lo que se traduce en la eliminacion de una nueva fuente de debilidades inherentes a las pavimentaciones de tipo ordinario. Para disminuir el rozamiento sobre la base se coloca una capa reduc-
tora de rozamientos entre la parte superior de la base y el firme. Esta capa reductora consta de una tongada de arena cubierta con papel de construcción o, lo que es igual, hojas de politileno.

Como sucede en otras pavimentaciones, las tensiones en los bordes libres entre juntas son de mayor consideración que las que se observan en el interior del trozo limitado entre dos bordes. Por esta razón, el pavimento de la Base de Biggs se proyectó de tal forma que en los bordes de cada uno de los tres trozos de 152 m de longitud, se soportaron sobre vigas de hormigón armado. Estas vigas se extienden unos $3 \mathrm{~m}$ más allá del borde de cada trozo. Entre trozos se dejó espacio suficiente para poder tesar todos los cables de la armadura. 


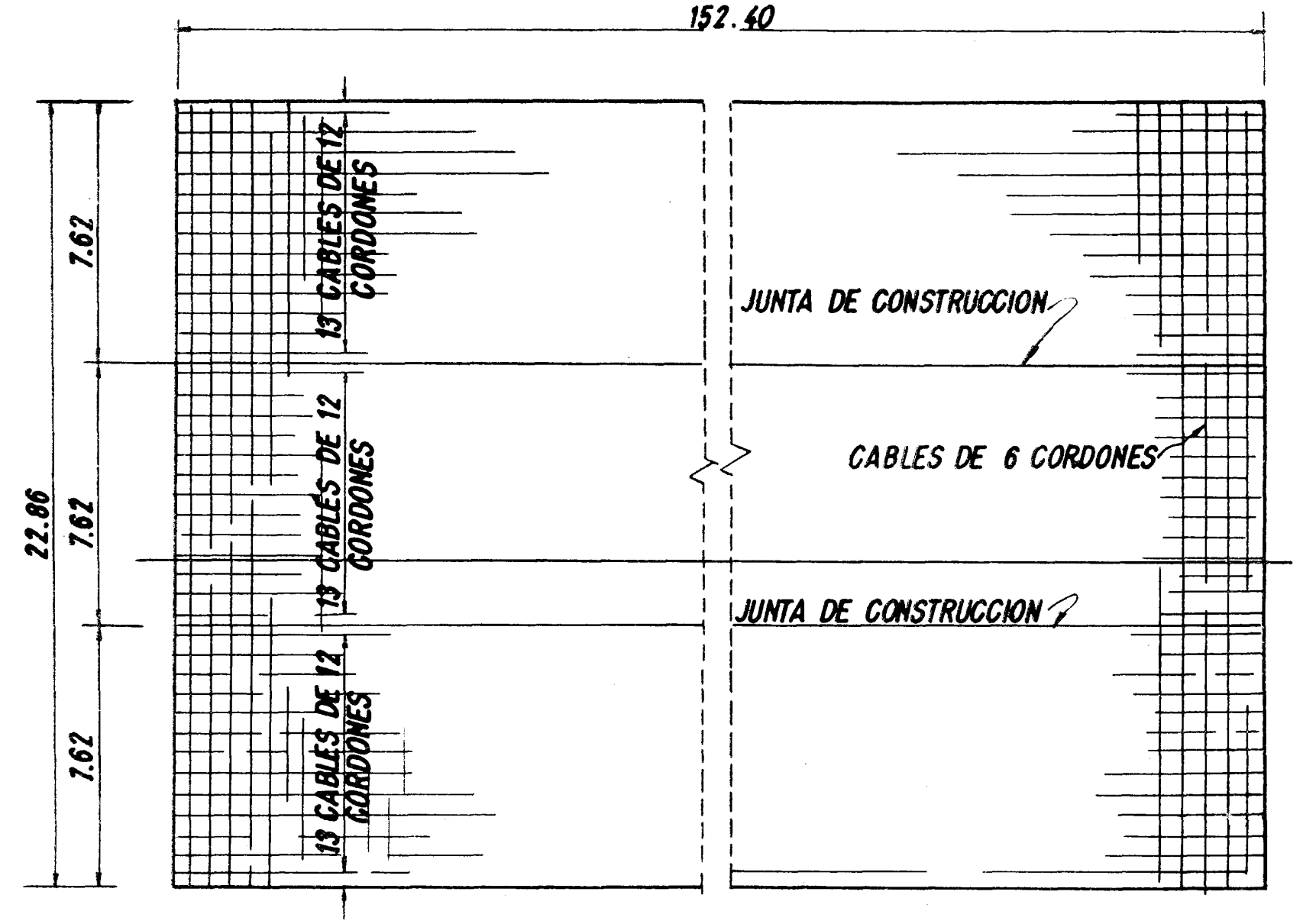

Plan general de construcción, inyecciones y anclajes.

Después de determinar las tensiones esperadas de las cargas dinámicas, el alabeo debido a la temperatura y rozamiento en la base, y considerando las tensiones debidas a la flexión del hormigón comprimido a $45 \mathrm{~kg} / \mathrm{cm} 2$, la tensión de pretensado calculada resultó ser de $24,50 \mathrm{~kg} / \mathrm{cm}^{2}$ en la dirección longitudinal y $12,30 \mathrm{~kg} / \mathrm{cm}^{2}$ en la transversal.

Para el cálculo del acero necesario para que el pretensado lograse un esfuerzo capaz de las tensiones antes mena en enta las pérdidas debidas, después de tesar, al acortamiento elástico del acero, deformación lenta, retracción del hormigón, relajación del acero y el rozamiento entre cables y conductos.

Con objeto de poder tomar en consideración todos los tipos de cables de acero, la elección del equipo de tesado que debía emplearse se dejó al libre arbitrio del contratista.

Se eligió alambre de alta resistencia a tracción, de $6,5 \mathrm{~mm}$ de diámetro, provisto de bulbo en su extremidad Cada uno de los trozos de $22,8 \mathrm{~m}$ de anchura y $472 \mathrm{~m}$ de longitud necesitó 39 cables de 12 alambres para el pretensado longitudinal. Estos cables se alojaron en tubos flexibles de acero, y se colocaron a una profundidad media del espesor del pavimiento o losa. Además, cada trozo lleva 223 cables transversales colocados inmediatamente debajo de los longitudinales, extendiéndose en toda la anchura de la losa. Los cables transversales están trenzados con 6 alambres de $6,5 \mathrm{~mm}$ de diámetro y se han alojado en tubos rigidos de acero. En cada extremidad de la cables se solidarizó una placa de acero para distribuir la carga concentrada en el cable al hormigón. En las os on en hormigón, con objeto de resistir los esfuerzos debidos a la gran presión que ejercen las placas de distribución de carga.

\section{Construcción}

La pista de rodadura $T-3$ se construyó, originalmente, de hormigón asfáltico colocado sobre una base de suelo-cemento. La parte central de esta pavimentación se quitó para poder construir la parte reforzada de la nueva pista de rodadura. 


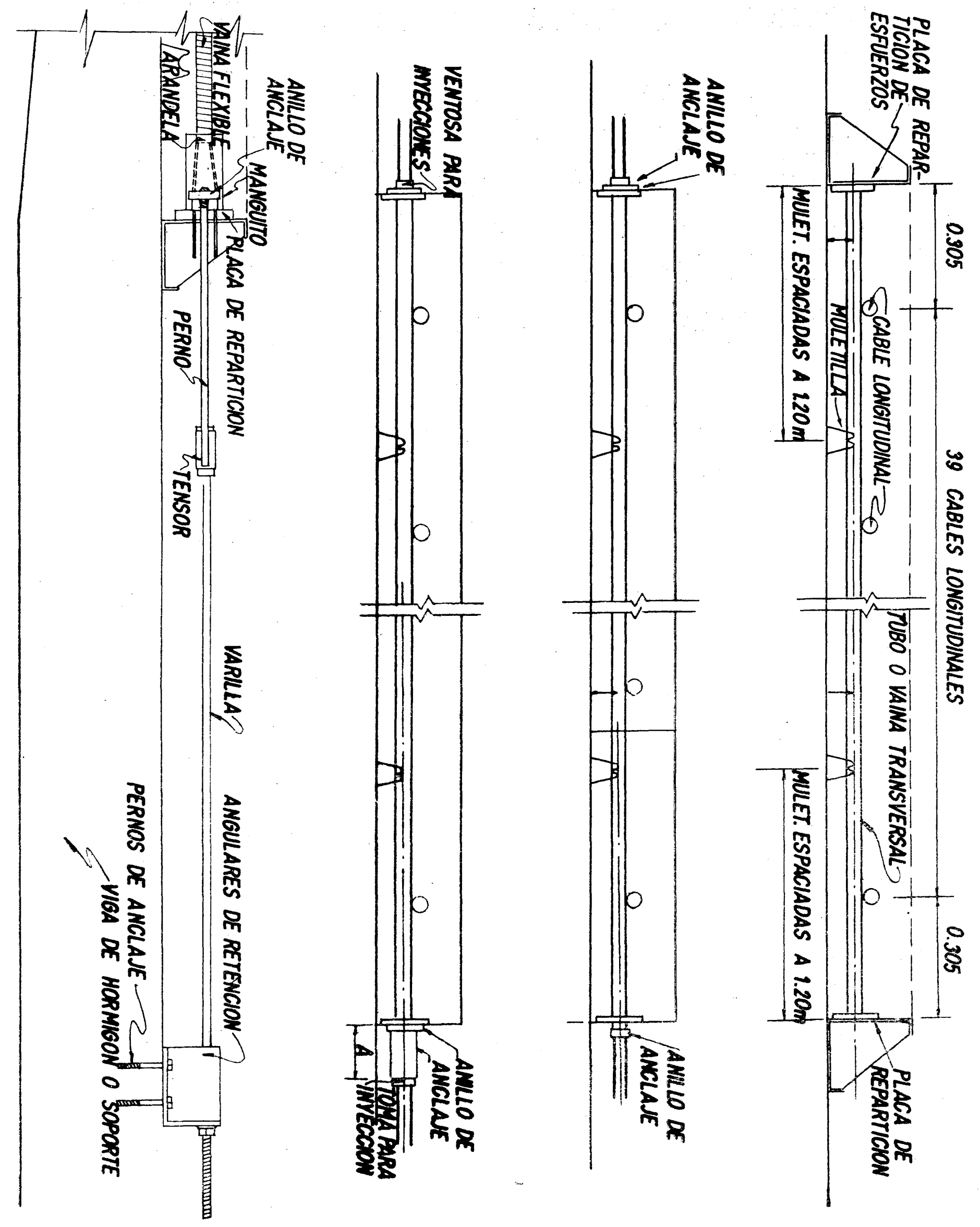



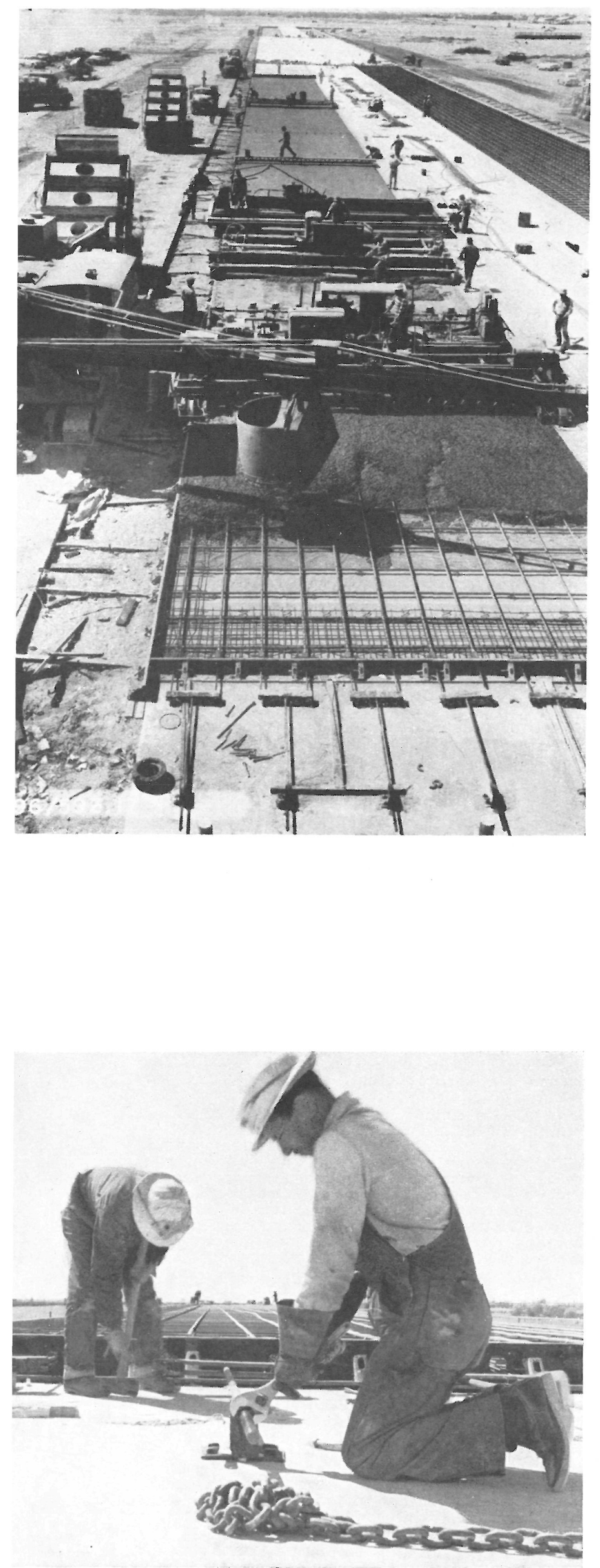

Hormigonado de la pista.

Tesado de los cables longitudinales.

La base del firme en todo el pavimento de la nueva pista era el mismo. Consiste en una capa de grava, de $15 \mathrm{~cm}$ de espesor, consolidada al 100 por 100 según las normas modificadas del AASHO sobre 2 capas formando cimiento: la primera compactada al $97 \%$ y la segunda o inferior al $92 \%$, de acuerdo con las instrucciones antes indicadas.

Las cuatro vigas de hormigón armado colocadas en la parte inferior de los bordes de cada trozo se construyeron sobre la base del firme. Su superficie superior se alisó con una llana para disminuir el rozamiento entre las vigas y la parte inferior de la losa del pavimento de $22 \mathrm{~cm}$ de espesor. Cada uno de los tres trozos de $472 \mathrm{~m}$ de longitud se Cadruyeron mando bandas de $7,60 \mathrm{~m}$ de anchura.

Se realizó en primer lugar la banda central, a cuya terminación se empezo la construccion simultanea de las dos laterales. Antes de montar los encofrados laterales de la losa se taladraron los costeros de los encofrados para poder colocar las piezas de plástico empleados para poportar los tubos transversales. piezas de plástico fijar piezas de plástico se de rellenar con gravilla y colocar la capa de arena y papel de politileno. A continuación se colocaron las armaduras corrugadas y los tubos o vainas de los cables $\mathrm{y}$, finalmente, se procedió a soportarlos por medio de muletillas. Los cables se colocaron en los tubos después de terminar las tres bandas. Los cables longitudinales, alojados en sus vainas metálicas, flexibles, se colocaron sobre los transversales.

Con objeto de mantener un trazado apropiado de los cables longitudinales durante el hormigonado, se procedió a tesarlos hasta conseguir una tensión de $700 \mathrm{~kg} / \mathrm{cm}^{2}$. Los anclajes provisionales para mantener a referida tensión se apoyaban en las vigas de las extremidades de cada trozo, donde se embebieron las piezas necesarias para cada anclaje. Estos anclajes son del tipo de tornillo y tuerca.

La ejecución de esta losa se realizó con un equipo auxiliar de pavimentadoras, esparcidoras, vibradoras, niveladoras, terminadoras y arrastre de arpilleras para el alisado final. Durante el hormigonado se obtenían probetas cilíndricas y viguetillas para someterlas a hormigón se logró con una batería de 12 vibradores montados sobre un bastidor móvil.

Las agujas de los vibradores penetraban hasta unos $5 \mathrm{~cm}$ de la parte inferior de la losa, y las vibradoras se colocaban de tal forma que se podían elevar al pasar sobre los tubos que alojan los cables transversales de pretensado, evitando así todo desperfecto a estos elementos. Complementando a estos equipos mecanicos de vibracion, se emplearon vibradores a mano para las zonas laterales y para las extremidades próximas a los bordes de los trozos donde se habían colocado las placas de reparto de esfuerzos concentrados en los cables.

El curado de la losa se llevó a cabo empleando mantos de algodón cargados de humedad, que cubrían la losa durante las primeras 30 ó 36 horas después de colocado el hormigón. A esta operación, seguía la colocación de una película de un pigmento que llevaba disuelto un compuesto para curado.

Durante la primera noche después de haber hormigonado la banda central, curada siguiendo los procedimientos ordinarios, aparecieron tres grietas, aproximadamente en el centro y en las terceras partes. Una de estas grietas apareció a unos $33 \mathrm{~m}$ de la extremidad, pero se cerró después de aplicar un pretensado parcial inicial de $7 \mathrm{~kg} / \mathrm{cm}^{2}$ al día siguiente. Al hormigonar las dos bandas laterales, las dos grieta aún abiertas en la banda central, experimentaron una especie de emigración hacia las bandas laterauna especie de emigracion hacia las bandas laterales. La temperatura del hormigon en el centro del espesor de las losas de estas bandas experimentó un descenso de $19^{\circ} \mathrm{C}$ durante la primera noche. 

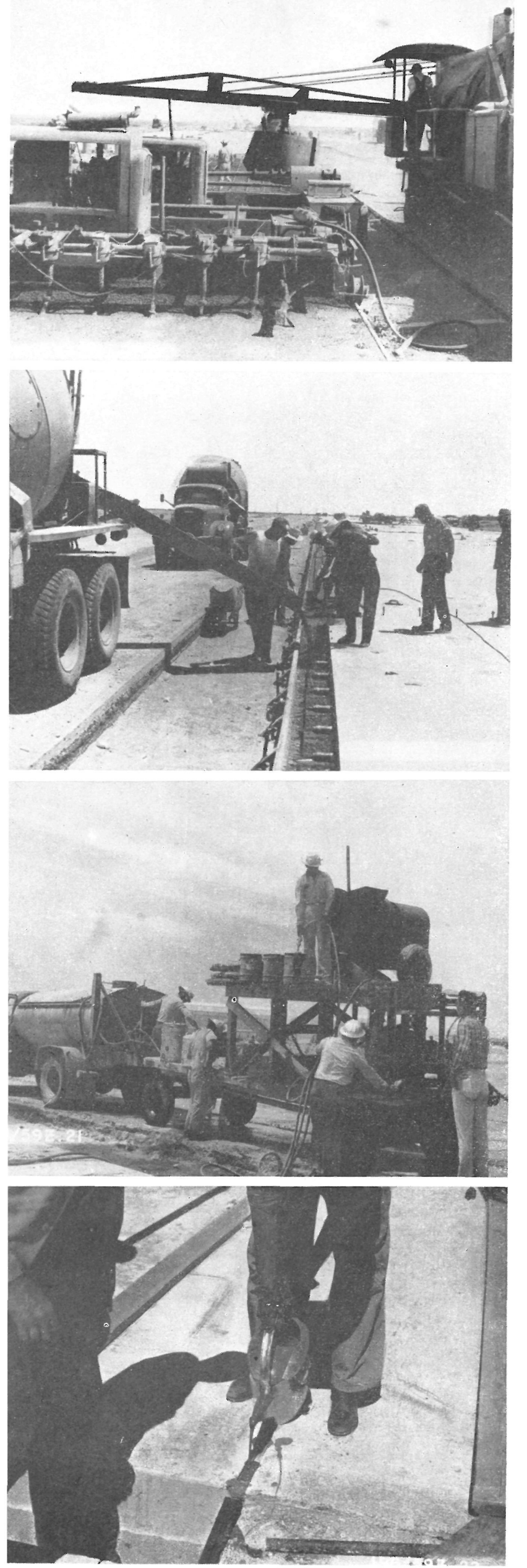

Batería de vibradores mecánicos.

Banda o margen de hormigón pobre.

Equipo de inyecciones.

Sellado de una junta de dilatación.

La banda central del segundo trozo se curó siguiendo los mismos procedimientos que en el primero, y aparecieron dos grietas transversales arbitrarias durante la primera noche. La diferencia de temperaturas durante este período fue de $14^{\circ} \mathrm{C}$. tensión de pretensado de $10,5 \mathrm{~kg} / \mathrm{cm}^{2}$ permitió cerrar prácticamente la grieta situada en la tercera parte prácticamente la grieta situada en la tercera parte de esta banda; sin embargo, la grieta de la proximidaterales de este trozo se curaron de igual manera que la central, pero con una recubrición adicional con mantos de algodón impermeabilizados con papel de construcción. Sólo la grieta de la banda central migró hacia las dos bandas laterales. Fn el tercer trozo se empleó el manto de algodón impermeabilizado en las empleó el manto de algodón impermeabilizado en las noche, el descenso de temperatura con este tipo de noche, el descenso de

El esfuerzo de pretensado aplicado preliminarmente se aflojó después de haber fraguado el hormigon. Un día después de haber hormigonado se aplicó una tensión de 7 a $10 \mathrm{~kg} / \mathrm{cm}^{2}$. Tan pronto como el hormigón de las tres bandas de un trozo determinado había adquirido suficiente resistencia, se aplicó la tensión final a todo el pavimento. Debido al rozamiento desarrollado entre cables y vainas, se procedió a tesar arrollado entre cables y vainas, se procedió a tesar partiendo de las dos extremidades simultáneamente. Los cables trans
una extremidad.

Después de terminar todas las operaciones del pretensado, se procedió a hormigonar con hormigón pobre las márgenes laterales longitudinales en una anchura de $38 \mathrm{~cm}$. También se rellenó de hormigón armado el espacio entre trozos. Las márgenes laterales tienen un espesor de $18 \mathrm{~cm}$ y se han recubierto con una capa de $5 \mathrm{~cm}$ de hormigón asfáltico.

Terminado el hormigonado se procedió a inyectar una lechada de cemento. La lechada consistió en una disolución o lechada de unos 20 litros de agua por saco de cemento, a la que se le añadió un compuesto retardador de fraguado $\mathrm{y}$ atenuador de la retracción. Antes ción empezó por la parte más baja hasta que da aparecía por la otra extremidad, o por ventosas practicadas entre los extremos procediendo, en este caso, por trozos sucesivos hasta llegar al final del trozo total. Aunque se pudo inyectar un trozo de $120 \mathrm{~m}$ de longitud a partir de un solo sitio, se comprobó que era mejor inyectar entre ventosas espaciadas a $30 \mathrm{~m}$ en los tubos longitudinales. La presión máxima de inyección fue de $10,5 \mathrm{~kg} / \mathrm{cm}^{2}$.

Después de terminar las inyecciones se formaron dos juntas de dilatación, una en cada extremidad de la pista pretensada. Cada una de estas juntas tenía una luz de $40 \mathrm{~mm}$ en el momento de su formación. Estas juntas consisten en un polisulfuro de caucho aplicado en forma de sellado en la parte inferior y superior de los $23 \mathrm{~cm}$ de espesor, rellenando el espacio hueco entre estos dos sellados con espuma de poliuretano de baja densidad. Además de estas dos juntas de dilatación, se formaron dos pseudojuntas, serradas en la parte central del relleno de hor entre trozos, practicadas tan pronto como el hormigón había fraguado suficientemente y no producía desportillado la sierra.

La operación final de esta pavimentación pretensada consistió en reparar las grietas aisladas que habian aparecido. Estas grietas se festonearon para darles una luz de $30 \mathrm{~mm}$ y se sellaron con una resina "epoxy".

La pavimentación realizada en esta base es la primera de esta clase que se realiza en ese país, y es objeto de especial observación, ya que los espesores reducidos empleados es un factor de consideración para el futuro. También se cree aportan una economía notable en los gastos de conservación.

Aunque el coste inicial es una tercera parte superior al de hormigón ordinario, se confía poder trabajar a precios de competencia actual. 